\title{
Corela
}

Cognition, représentation, langage

HS-10 | 2012

Paramétrer le sens ? Études de cas

\section{La relation inter-énonciative et le marquage syntaxique des relations de cause : étude contrastive anglais-français.}

Agnès Leroux

\section{(2) OpenEdition}

\section{Journals}

Édition électronique

URL : http://journals.openedition.org/corela/2429

DOI : $10.4000 /$ corela.2429

ISSN : 1638-573X

Éditeur

Cercle linguistique du Centre et de l'Ouest - CerLICO

\section{Référence électronique}

Agnès Leroux, «La relation inter-énonciative et le marquage syntaxique des relations de cause : étude contrastive anglais-français. », Corela [En ligne], HS-10 | 2012, mis en ligne le 30 janvier 2012, consulté le 19 avril 2019. URL : http://journals.openedition.org/corela/2429 ; DOI : 10.4000/corela.2429

Ce document a été généré automatiquement le 19 avril 2019

\section{(c) (i) (2)(2)}

Corela - cognition, représentation, langage est mis à disposition selon les termes de la licence Creative Commons Attribution - Pas d'Utilisation Commerciale - Partage dans les Mêmes Conditions 4.0 International. 


\title{
La relation inter-énonciative et le marquage syntaxique des relations de cause : étude contrastive anglais- français.
}

\author{
Agnès Leroux
}

\section{Introduction}

1 Cette étude présente le bilan d'une suite d'études contrastives anglais/français (Leroux 2009,2010 ) qui nous ont amenée à nous interroger sur la construction du sens en fonction de la relation entre énonciateur et co-énonciateur. Les conclusions de ces différentes analyses montrent que la relation inter-énonciative associable à différents genres de discours influe de façon significative sur le marquage linguistique d'un phénomène.

2 Nous nous sommes intéressée au marquage de la relation de cause, et plus particulièrement de la relation d'effet à cause, sur des corpus de nature et de langues différentes, ayant en effet mené plusieurs études contrastives anglais/français sur ce type de relation sémantique sur des corpus comparables (aucun texte n'est traduit) constitués dans des genres différents : la presse quotidienne, des blogs écrits par des journalistes, et des exemples de discours politiques nationalistes.

Il s'agit donc de croiser plusieurs paramètres incidents au choix de marquer explicitement ou non une relation causale, et à la construction du sens de cette relation dans l'énoncé : langue, type de discours et de relations inter-énonciatives, nature et portée de la relation causale. Il est apparu que l'utilisation d'un marqueur dépend en partie du type de relation énonciateur/co-énonciateur dans laquelle est produit l'énoncé à valeur causale, et que l'utilisation ou la non-utilisation d'un marqueur ne correspond pas aux mêmes configurations causales selon la relation inter-énonciative en anglais et en français. Notre ultime question est la suivante : quel sens construisent l'asyndète d'une 
part et le marquage syntaxique explicite d'autre part, si l'on considère que la plus grande partie des relations de cause est exprimée sans marqueur?

Cette étude comporte trois mouvements :

- Une définition de la relation inter-énonciative telle que nous l'envisageons et une description des caractéristiques significatives des différentes relations inter-énonciative dans chaque corpus utilisé.

- Une présentation globale des résultats avec des tableaux contrastifs.

- Une analyse plus précise d'un marquage causal dans les discours politiques à tendance nationaliste : la relative.

4 Le corpus sur lequel nous avons fondé cette étude a fait l'objet d'un relevé manuel. Il est en effet impossible de pratiquer des relevés électroniques au sein d'un corpus dont une grande partie des exemples est constituée d'énoncés sans marques syntaxiques distinctes. Les chiffres que nous produisons ici sont donc à considérer représentant des tendances plutôt que des valeurs absolues.

5 Nos exemples journalistiques ont été prélevés sur des quotidiens français et anglais, ceux des blogs, sur des blogs de journalistes anglophones et francophones, et ceux relevant du discours nationaliste sont extraits des blogs de Nick Griffin et de Jean-Marie Le Pen, sur lesquels sont retranscrits leurs discours et différentes interventions. Chaque partie du corpus représente environ 200 énoncés.

\section{Définition de la relation inter-énonciative}

\section{I.1. Types de discours et relations inter-énonciatives}

6 Pour définir la relation inter-énonciative propre à chaque type de production étudiée dans notre corpus, nous réfèrerons dans un premier temps à l'appareil formel de l'énonciation, utilisé dans la Théorie des Opérations Prédicatives et Enonciatives (désormais TOPE) pour décrire la détermination des énoncés. Celui-ci se compose : d'un énonciateur (aussi noté $\mathrm{S}_{0}$ ), instance abstraite représentant toute source du discours (l'instance concrète étant le locuteur), d'un co-énonciateur, représentant toute représentation d'autrui dans le discours, y compris le destinataire du discours, d'un contenu et d'un instant d'énonciation, espace-temps (aussi noté $\mathrm{T}_{0}$ ) de la production du discours. La détermination d'un énoncé est le résultat de la conjonction de ces quatre principaux paramètres. Nous nous concentrerons plus précisément sur la relation intersubjective entre énonciateur et co-énonciateur qui peuvent être, une fois le discours en cours de production dans un dialogue, des instances concrètes et non-plus abstraites du discours, et peuvent alors être identifiés et désignés par les termes locuteur et colocuteur.

\section{I.1.1. Trois relations inter-énonciatives particulières}

7 Dans le corpus journalistique issu de la presse quotidienne, la relation inter-énonciative est différée et à sens unique : en effet, l'énonciateur, en l'occurrence le journaliste, n'a aucun contact et donc aucun échange avec le co-énonciateur potentiel qu'est le lecteur (comme pour une communication épistolaire). En outre, la lecture se fait plusieurs heures après l'écriture, posant un problème de repérage entre moment d'écriture, référence aux événements, et moment de la lecture. La relation inter-énonciative n'est pas ancrée dans 
la situation origine de l'énonciation ( $\left.\mathrm{Sit}_{0}\right)$, et le co-énonciateur destinataire n'a pas statut d'énonciateur potentiel.

Dans les blogs, ici de journalistes, la relation inter-énonciative est également différée mais elle peut s'instaurer dans les deux sens et voir énonciateur et co-énonciateur inverser les rôles, puisqu'une intervention du lecteur est possible. La communication et l'échange de rôles ne se fait cependant pas de façon instantanée.

9 Avec les discours politiques, la relation inter-énonciative est directe en $\mathrm{Sit}_{0}$, puisque l'énonciateur s'adresse directement, sans délai, à son auditoire, qui lui tient lieu de coénonciateur. Mais elle est à sens unique puisque la réponse, du moins verbale, est rarement possible et n'est d'ailleurs pas envisagée. L'assentiment est la réponse attendue. Comme dans la presseécrite, le co-énonciateur n'a pas statut d'énonciateur producteur potentiel.

10 La façon dont se construisent ces trois types de relations a une incidence selon nous sur la façon dont s'expriment les relations de cause, surtout dans les parties argumentatives du discours.

\section{I.1.2. Deux langues différentes : anglais et français}

11 Chaque étude a été menée séparément à l'origine et de façon contrastive. Notre objectif était d'observer les deux langues de façon indépendante, de façon à éviter le plus possible que l'observation de l'une n'influe sur l'observation de l'autre. Nous avons donc travaillé sur corpus comparables, c'est-à-dire sur deux corpus en langue source : l'un anglais et l'autre français. Le corpus journalistique est issu de la presse quotidienne, dite 'presse de qualité', le corpus de blogs est relevé sur Internet à partir des blogs personnels de journalistes de la presse quotidienne, et le corpus de discours nationalistes est également relevé sur Internet, sur les sites des partis nationalistes où sont transcrits les discours. Cependant, quand l'observation ne se fait pas à partir de traductions et donc d'équivalence directe d'un énoncé avec un autre, il est nécessaire de référer à des critères d'observation stables qui permettent d'analyser les deux corpus parallèles.

Notre codage visait à relever la présence ou l'absence de marqueurs syntaxiques lorsque deux propositions sont liées par une relation d'effet à cause. Les critères de relevé sont donc à la fois sémantiques et syntaxiques. Dans un souci de cohérence, nous n'avons observé que des énoncés dans lesquels la première proposition était expliquée par la seconde, ou relation d'effet à cause. Nous obtenons quatre catégories :

- Justification ou explication de la survenue d'un événement par un autre événement ou une propriété (critères essentiellement afférents au paramètre T)

- Explication de la survenue d'un événement par une hypothèse propre au journal ou à la source énonciative(T/S)

- Explication d'un jugement propre au journal ou à la source énonciative par un événement ou une propriété (S/T)

- Explication d'un jugement propre au journal, ou à la source énonciative par un autre jugement ou évaluation (s/S).

Nous avons schématisé nos énoncés selon l'exemple suivant :

(1) Parce que l'an dernier, il a connu une déception à l'élection présidentielle et un grave échec aux élections législatives, certains, prenant leurs désirs pour des réalités et les vessies pour des lanternes, ont claironné qu'il était mort. JMLP. 
13 Où nous relevons la structure $\mathrm{P} P$ parce que $\mathrm{q}$ : Certains ont claironné qu'il était mort $(\mathrm{p})$ parce qu'il avait connu une déception et un grave échec aux élections présidentielles (q). Et dans cette structure :

$\mathrm{p}$ est structurée au niveau prédicatif en une première relation prédicative, désormais RP1 : <personne -claironner que <...>>

$\mathrm{q}$ est structurée en une deuxième relation prédicative, désormais RP2: <Front National - connaître grave échec>

14 Voici, dans le Tableau 1 ci-dessous, un résumé à partir d'exemples typiques illustrant les quatre configurations argumentatives énumérées ci-dessus :

Tableau 1 : Schématisation des configurations argumentatives

\begin{tabular}{|l|l|l|}
\hline Configuration argumentative & EFFET & CAUSE \\
\hline $\begin{array}{l}\text { Explication factuelle portant sur un } \\
\text { fait }\end{array}$ & L'avion a disparu de nos radars & $\begin{array}{l}\text { il est tombé dans } \\
\text { l'Atlantique }\end{array}$ \\
\hline Explication modale portant sur un fait & L'avion a disparu des radars & $\begin{array}{l}\text { il serait tombé dans } \\
\text { l'Atlantique }\end{array}$ \\
\hline $\begin{array}{l}\text { Explication factuelle portant sur une } \\
\text { modalité }\end{array}$ & L'avion serait tombé & iln'en reste aucune trace \\
\hline $\begin{array}{l}\text { Explication modale portant sur une } \\
\text { modalité }\end{array}$ & $\begin{array}{l}\text { L'avion serait tombé dans } \\
\text { l'Atlantique }\end{array}$ & $\begin{array}{l}\text { peut-être y a-t-il eu une } \\
\text { tempête }\end{array}$ \\
\hline
\end{tabular}

Voici dans le Tableau 2 une illustration faite d'exemples authentiques :

Tableau 2 : Illustration des configurations argumentatives

\begin{tabular}{|c|c|}
\hline $\begin{array}{l}\text { Configuration } \\
\text { argumentative }\end{array}$ & Exemples tirés de la presse quotidienne \\
\hline $\begin{array}{l}\text { 1.Explication } \\
\text { factuelle portant sur } \\
\text { un fait }\end{array}$ & $\begin{array}{l}\text { Par ailleurs, l'inquiétude grandit face à la menace d'attaques chimiques. Un } \\
\text { attentat au chlore a fait deux morts et intoxiqué } 350 \text { personnes près de Falloujah. }\end{array}$ \\
\hline $\begin{array}{l}\text { 2.Explication modale } \\
\text { portant sur un fait }\end{array}$ & $\begin{array}{l}\text { Pas un mot en revanche, sur le nucléaire iranien, dont il est plus que probable } \\
\text { qu'il a été question. Ni Londres, ni Washington ne veulent donner l'impression } \\
\text { qu'un quelconque marchandage est d'actualité au moment où une troisième } \\
\text { vague de sanctions contre Téhéran est à l'étude aux Nations Unies sur initiative } \\
\text { des pays occidentaux. }\end{array}$ \\
\hline $\begin{array}{l}\text { 3.Explication } \\
\text { factuelle portant sur } \\
\text { une modalité }\end{array}$ & $\begin{array}{l}\text { Les néerlandais ont été choqués d'apprendre que plus de } 300 \text { millions d'euros ont } \\
\text { été investis en } 2006 \text { par leurs fonds de pension dans des entreprises nord- } \\
\text { américaines fabriquant des bombes à fragmentation et des mines antipersonnel. } \\
\text { Ces armes font, en effet, l'objet de traités internationaux visant à les éradiquer, } \\
\text { rappelle le quotidien De Volksrant. }\end{array}$ \\
\hline
\end{tabular}


4.Explication modale

portant sur une

modalité

Tous ces partis doivent désormais se consacrer aux législatives car il y va

désormais de leur survie.

Nous introduisons pour chaque catégorie de discours des données quantitatives qu'il faut prendre avec beaucoup de prudence dans la mesure où les corpus sur lesquels sont fondés ces études ont fait l'objet d'un relevé manuel, donc forcément limité (entre 100 et 250 énoncés selon les corpus).

\section{Résultats contrastifs français/anglais par type de discours}

\section{II.1. Presse quotidienne}

17 Au sein d'un même type de discours, ici la presse quotidienne, on observe des tendances différentes selon les langues, quant au choix de marquer ou non le lien explicatif par un connecteur, selon laconfiguration explicative. Ces tendances sont illustrées ci-dessous par quelques exemples parmi les énoncés que nous avons mis en regard:

\section{Conf 1 : Explication factuelle portant sur un fait}

En anglais :

(2) When Amanda Knox looked into an Italian documentary film-maker's camera and recited the "to be or not to be" speech from Hamlet, the select few who viewed the film heralded it as a stellar performance. It was the debut no one saw, however, because authorities blocked the documentary film about women in prison once they discovered Ms Knox was the star.(The Guardian International)

18 Nous nous intéressons à la partie d'énoncé constituée de deux propositions structurées selon les deux relations prédicatives suivantes: RP1 < people - see - debut > et RP2 < authority - block - film >. La relation de cause entre les deux propositions est marquée >par because. Because n'est pas le seul marqueur que nous ayons >relevé dans cette catégorie : nous avons également since , >des participe passés, des participes présents, des locutions incluant le nom cause, et des relatives. Dans cette configuration causale, une proportion importante d'énoncés contient un marqueur en anglais.

En français :

(3) Par ailleurs, l'inquiétude grandit face à la menace d'attaques chimiques. Un attentat au chlore a fait deux morts et intoxiqué 350 personnes près de Falloujah. (Libération)

19 Ici nous avons deux phrases structurées selon les relations prédicatives suivantes: RP1 <inquiétude - grandir > et RP2 < attentat - faire - mort >. La relation entre les deux éléments de la relation causale n'est marquée que par un signe de ponctuation. Ceci est le cas le plus fréquemment relevé dans cette configuration causale (numérotée 1) en français, même si nous avons également relevé des marqueurs de cause : car, en effet, et quelques occurrences de parce que.

\section{Conf. 2 : Explication modale portant sur un fait}

En anglais : 
(4) No one before or since has had the dexterity to make such transparent child's play of Bach's severest contrapuntal puzzles. That he played these pieces at such blinding speeds was not necessarily because he should have; I think he just wanted us to know that he could. (The Herald Tribune)

En français :

(5) Pas un mot en revanche, sur le nucléaire iranien, dont il est plus que probable qu'il a été question. $\emptyset \mathrm{Ni}$ Londres, ni Washington ne veulent donner l'impression qu'un quelconque marchandage est d'actualité au moment où une troisième vague de sanctions contre Téhéran est à l'étude aux Nations Unies sur initiative des pays occidentaux. (Libération)

En (4), la relation d'effet à cause est la suivante : RP1 <musician - play fast >expliquée par RP2 <musician -want - <people - know <musician - play -fast>>> Les deux relations sont liées une première fois, dans une relation négative, par because, ce qui est le cas le plus fréquemment relevé en configuration causale 2 en anglais. En revanche, nous constatons qu'en (5), RP1 < chef d'état - parler - nucléaire iranien > n'est liée par aucun marqueur à RP2<Londres et Washington - vouloir - ... > .

\section{Conf. 3 : Explication factuelle portant sur une modalité}

En anglais :

(6) At least some of the killings appear to have been assassinations. $\varnothing$ On Monday a supreme court official with close ties to Mr Chaudhry, Syed Hammad Raza, was shot dead at his home in Islamabad. (The Guardian Weekly)

En français :

(7) Rien dans la déclaration liminaire à la conférence de presse prononcée par M. Ahmadinejad, ne permettait pourtant d'espérer une libération imminente des quinze marins, dont une femme Faye Turney. Pendant près d'une heure, en effet, le président iranien s'est livré à un aperçu de l'histoire de la région depuis le XXème siècle, couvrant d'opprobre l'Occident, en particulier la Grande Bretagne et les Etats-Unis. (Le Monde)

21 En (6), et (7), nous constatons que la tendance s'inverse : le marqueur de cause est plus fréquemment relevé en français qu'en anglais. Nous avons une asyndète en 14 et en effet en 15.

\section{Conf. 4 : Explication modale portant sur une modalité}

En anglais :

(8) so why did the voters narrowly decide to turf out Goran Persson, their prime minister for ten years, in favour of Frederik Reinfeldt, leader of the moderate party and of the four-party opposition alliance ?ø there are three answers. the first is the peril of long incumbency. Anybody seeking a fresh term after ten years in office had better be an adept salesman. (TheHerald Tribune)

En français :

(9) Tous ces partis doivent désormais se consacrer aux législatives car il y va désormais de leur survie. (Libération)

L'inversion de tendance se confirme avec la configuration causale 4 comme l'illustrent les énoncés (8) et (9) : les marqueurs de cause sont largement plus fréquemment relevés en français qu'en anglais. Ces tendances observées dans l'ensemble de notre corpus sont synthétisées dans le tableau ci-dessous: les pourcentages concernent la proportion d'utilisation d'un marqueur par rapport à sa non-utilisation, le marqueur mentionné dans la grille est le plus fréquent. 
Utilisation des marqueurs de cause dans la presse quotidienne :

Tableau 3 : Utilisation des marqueurs de cause dans la presse quotidienne

\begin{tabular}{|l|l|l|}
\hline Configuration argumentative & anglais & français \\
\hline 1. Explication factuelle portant sur un fait & $\begin{array}{l}50 \% \\
\text { Because }\end{array}$ & $11 \%$ \\
\hline 2. Explication modale portant sur un fait & $\begin{array}{l}39 \% \\
\text { Because }\end{array}$ & $16 \%$ \\
\hline 3. Explication factuelle portant sur une modalité & $18 \%$ & $\begin{array}{l}22 \% \\
\text { En effet }\end{array}$ \\
\hline 4. Explication modale portant sur une modalité & Proche 0 \% & $\begin{array}{l}34 \% \\
\text { Car }\end{array}$ \\
\hline
\end{tabular}

Suite à ces données quantitatives, nous constatons une utilisation inverse des marqueurs de cause dans chaque configuration causale. Nous pourrions même dire que nous obtenons un tableau en miroir de la configuration causale 1 à la configuration causale 4 .

Il semblerait donc que la construction du sens d'une relation de cause ne se fasse pas de la même façon en anglais qu'en français. Du moins ici dans les journaux quotidiens de qualité. Plus il s'agit d'exprimer un point de vue ou de donner un argument, en anglais, moins le recours à un marqueur semble nécessaire. Et inversement en français : plus l'explication est modale, plus le marqueur est utilisé.

\section{II.2. Blogs de journalistes}

Voici quelques exemples représentatifs des relevés effectués sur des blogs de journalistes.

\section{Configuration 1}

Anglais :

(10) But after three days in a massive steel and glass hangar on the edge of this bustling city-state, I can see the logic of meeting here. I also have a better sense of the health of a sector being buffeted by the chill winds of banking crises and globalslowdown.

Abu Dhabi, with $8 \%$ of the world's proven oil reserves, is hosting this summit because its crown prince likes renewables. (Blog de Terry Mc Alister)

Français :

(11) Lorsqu'une personnalité fait une déclaration tonitruante sur un plateau de télévision, lorsqu'une chaîne d'info en continu diffuse des images d'une catastrophe, ou l'interpellation du président par un marin-pêcheur, l'internaute a pris l'habitude d'aller sur Internet pour en voir un extrait, et en débattre dans les commentaires ou les forums.

Et il va chez FullHdReady parce que les médias traditionnels ne proposent pas ce service d'information. (blog de B. Raphaëlle)

Nous voyons que les énoncés (18) et (19), représentatifs de cette partie de notre corpus, sont construits de la même façon: RP1 marqueur RP2. Les marqueurs dans cette partie 
sont divers mais il y a une prévalence du marqueur because en anglais et de parce que en français.

\section{Configuration 2}

Anglais :

(12)For 24 hours, Noddy implies, Santa hides the corkscrew, because he has reindeer to drive and chimneys down which he's loth to fall. (It would alert the children.) (Blog de Jon Canter)

Français :

(13) A une exception près, je n'ai jamais voulu parler sur ce blog de mon expérience au Post.fr. D'abord parce qu'il s'agit d'un blog personnel dont l'objectif est d'ouvrir des conversations sur l'évolution des médias et du journalisme, et qu'il est toujours compliqué de parler de son propre média sans donner l'impression de faire de l'autopromotion. (Blog de B. Raphaëlle)

En configuration causale 2 (un fait expliqué par une modalité), nous retrouvons le parallèle entre les deux langues. Les constructions sont à peu de choses près équivalentes.

\section{Configuration 3}

Anglais :

(14) But Graham Appleton of the British Trust for Ornithology urges anyone who does see a barn owl not to feel disappointed. Any sighting - of any species of owl is valuable, especially to the BTO, which is currently carrying out the biggest ever survey of Britain's birds with the help of thousands of ordinary birders. (Blog de Stephen Moss)

Français :

(15) Le Washington Post poursuit néanmoins l'expérience, et il a raison. L'intérêt de ce type d'expérience n'est pas tant de faire du chiffre tout de suite que d'expérimenter afin de conquérir un territoire encore largement inconnu.(Blog de B. Raphaëlle)

L'énoncé (14) est construit d'une façon particulière, construction que nous avons fréquemment relevée dans une autre partie de notre corpus : le discours nationaliste. Le lien d'effet à cause entre RP1 [ sighting of barn owl - be - valuable ...] et RP2 [ BTO - carry out - survey ] est établi avec une relative, dite 'relative explicative'. Cette structure n'est pas explicitement un marqueur de cause, mais il ne s'agit pas d'une asyndète, puisque les propositions sont liées par une opération de subordination. Nous avons cependant moins de marqueurs explicites de cause dans cette partie du corpus en anglais que dans les configurations 1 et 2. En français également, conformément à l'énoncé cité en (15), l'utilisation de marqueurs est moindre.

\section{Configuration 4}

Anglais :

(16) We are those cop-out, fair-weather Jews that "real" Jews despise more than they do antisemites : the secular, cultural Jews, the amoral majority, the ones who want to have their bagel and eat it. (Blog de Jonathan Magolis)

Français :

(17) Ce qu'il ne dit pas, c'est que la récente déclaration de candidature de Valérie Pécresse (notre billet du 16 juillet) a changé la donne. Entre le candidat légitime d'un côté, la jeune et populaire ministre de l'autre, l'espace était devenu trop étroit. (Blog d'Alain Auffray) 
Tableau 4 : Présentation synthétique du classement des énoncés extraits des blogs de journalistes

\begin{tabular}{|c|c|c|}
\hline Configuration argumentative & Anglais & Français \\
\hline $\begin{array}{l}\text { 1.Explication factuelle portant } \\
\text { sur un fait }\end{array}$ & 50 \%marqueurs Because $35 \%$ & $\begin{array}{l}53 \% \text { marqueurs } \\
\text { car et parce que }\end{array}$ \\
\hline $\begin{array}{l}\text { 2.Explication modale portant } \\
\text { sur un fait }\end{array}$ & $\begin{array}{l}60 \% \text { marqueur , } 25 \% \text { because ou } \\
\text { forme de questions en RP1 }\end{array}$ & $\begin{array}{l}58 \% \text { marqueurs } \\
\text { Car, parce que, en effet, } \\
\text { quelques relatives }\end{array}$ \\
\hline $\begin{array}{l}\text { 3.Explication factuelle portant } \\
\text { sur une modalité }\end{array}$ & $30 \%$ marqueurs, relative & $50 \%$ marqueurs \\
\hline $\begin{array}{l}\text { 4.Explication modale portant } \\
\text { sur une modalité }\end{array}$ & $10 \%$ marqueurs & $-39 \%$ marqueurs \\
\hline
\end{tabular}

31 Ce tableau synthétique semble montrer que le marquage linguistique de la relation causale se fait à peu de choses près de la même façon en anglais et en français, même si les proportions ne sont pas exactement les mêmes. Les proportions sont très différentes de celles établies précédemment, et nous ne retrouvons pas la structure en miroir mise en évidence dans le tableau chiffré consacré à la partie journalistique du corpus. Cecisignifie que le type de discours est un paramètre pertinent dans la construction du sens causal. Selon qu'un journaliste s'exprime dans la presse écrite ou sur un blog internet, il n'utilise pas les marqueurs de cause de la même façon.

\section{II.3. Discours politiques nationalistes}

Le choix d'analyser les structures causales dans des discours politiques nationalistes nous fait passer non-seulement d'une catégorie écrite à une catégorie de discours oral mais en plus à un genre argumentatif très différent. Le locuteur a, de façon explicite, un message à faire passer. Il doit être convaincant. Les énoncés relevés dans les transcriptions de discours de Jean Marie Le Pen sont notés JMLP et ceux relevés dans les discours de Nick Griffin sont notés NG.

\section{Configuration argumentative 1}

En anglais :

(18) Increasingly our people are facing denial of service provision, failure to secure business contracts as well as poor job prospects as reverse discrimination excludes our people from the school room, workplace and boardroom. NG. 
Dans cet exemple, une relation d'effet à cause est instaurée entre RP1 <people - face denial > et RP2 < reverse discrimination - exclude - people >, marquée par as. As a ici une valeur proche de la concomitance. L'énonciateur asserte ainsi la valeur factuelle de la relation, comme s'il n'était pas responsable de la mise en relation causale. Nous relevons dans cette configuration beaucoup d'occurrence de as et de because.

En français :

(19) Parce que l'an dernier, il a connu une déception à l'élection présidentielle et un grave échec aux élections législatives, certains, prenant leurs désirs pour des réalités et les vessies pour des lanternes, ont claironné qu'il était mort. JMLP.

En (19) l'ordre d'effet à cause est marqué par parce que : RP1 <certain-claironner < FN - être - mort >> expliqué par RP2 <FN -connaître - échec >. Ce sont deux faits qui sont mis en relation par parce que. Parce que est le marqueur le plus caractéristique de cette configuration causale, mais nous avons relevé d'autres marqueurs et structures: utilisation d'un participe présent ou passé et quelques en effet. Il est très rare qu'il n'y ait pas de marqueur.

constatons donc une utilisation comparable des marqueurs dans cette configuration causale en anglais et en français. Nous ne nous intéresserons cependant pas à la correspondance des marqueurs entre eux, mais uniquement à leur présence ou absence, même s'il apparaît que la fréquence de chacun en fonction des types de discours et des configurations argumentatives pourrait constituer le sujet d'une étude.

\section{Configuration argumentative 2}

En anglais :

(20) The discrimination and the deprivation, the sense of alarm and of resentment, lies not with the immigrant population but with those among whom they have come and are still coming.

This is why to enact legislation of the kind before parliament at this moment is to risk throwing a match on to gunpowder. NG.

Pour bien comprendre la structure de cet énoncé, il est utile de restaurer la structure que évoquée jusqu'ici, c'est-à-dire la relation d'effet à cause :

$\left(20^{\prime}\right)$ To enact legislation of the kind before parliament at this moment is to risk throwing a match on to gunpowder because the discrimination and the deprivation, the sense of alarm and of resentment, lies not with the immigrant population but with those among whom they have come and are still coming.

En français :

(21) C'est cette politique suicidaire qui explique l'effondrement de notre commerce extérieur, parce que la politique dite de "l'euro fort" pénalise les exportations françaises, tandis que l'ouverture commerciale entraine l'invasion de nos marchés et la destruction de pans entiers de notre économie. JMLP.

Les deux marqueurs de cause utilisés dans ces exemples, largement relevés dans cette configuration, sont beaucoup moins présents dans les autres discours en configuration 2 . This is why est très peu utilisé dans les blogs et le discours journalistique anglais. En français, nous avons relevé d'autres marqueurs que parce que même si celui-ci est le plus fréquent: il est vrai, structuration de la relation de cause autour d'une question, car, parce que.

\section{Configuration argumentative 3}

En anglais : 
(22) I simply do not have the right to shrug my shoulders and think about something else. What he is saying and thinking - not throughout Great Britain perhaps, but in the areas that are already undergoing the total transformation to which there is no parallel in a thousand year of English history. NG.

En français :

(23) Sarkozy, malgré tout son talent de bonimenteur, aura donc beaucoup de mal à faire accepter et adopter des projets ou des propositions, même souhaitables , dont les autres ne voudraient pas. Tout simplement parce que nous ne montrons pas

l'exemple. JMLP.

Dans cette configuration, nous avons relevé quelques énoncés contenant des marqueurs de cause, en retenant tout de même une proportion moitié moindre en anglais. C'est la configuration argumentative pour laquelle nous trouvons le plus souvent une asyndète.

\section{Configuration argumentative 4}

En anglais :

(24) We will also clamp down on the flood of 'asylum seekers', all of whom are either bogus or can find refuge much nearer their home countries. NG.

Tableau 5 : Synthèse du classement des énoncés extraits des discours politiques nationalistes

\begin{tabular}{|l|l|l|}
\hline $\begin{array}{l}\text { Configuration } \\
\text { argumentative }\end{array}$ & Français & Anglais \\
\hline $\begin{array}{l}\text { Explication factuelle } \\
\text { portant sur un fait } \\
\text { configuration 1 }\end{array}$ & $\begin{array}{l}\text { Utilisation de marqueurs à } 60 \% .30 \% \\
\text { de participes présents et 1\% de } \\
\text { participes passés. } \\
\text { Asyndète quasi nulle. }\end{array}$ & $\begin{array}{l}\text { Utilisation de marqueurs à } \\
83 \% \text { because, as, after. }\end{array}$ \\
\hline $\begin{array}{l}\text { Explication factuelle } \\
\text { portant sur une modalité } \\
\text { configuration 2 }\end{array}$ & $\begin{array}{l}\text { Utilisation de marqueurs à 96\% } \\
\text { parce que, car et le verbe expliquer dans } \\
\text { différentes locutions verbales. }\end{array}$ & $\begin{array}{l}\text { Utilisation de marqueurs à } \\
80 \% \text {, because, as. }\end{array}$ \\
\hline
\end{tabular}




\begin{tabular}{|l|l|l|}
\hline $\begin{array}{l}\text { Explication modale } \\
\text { portant sur un fait } \\
\text { configuration 3 }\end{array}$ & $\begin{array}{l}\text { Utilisation de marqueurs à 30\% } \\
\text { puisque, en effet. }\end{array}$ & $\begin{array}{l}\text { Utilisation de marqueurs à } \\
17 \% \\
\text { because }\end{array}$ \\
\hline $\begin{array}{l}\text { Explication modale } \\
\text { portant sur une modalité } \\
\text { configuration } 4\end{array}$ & $\begin{array}{l}\text { Utilisation de marqueurs à 40\% } \\
\text { puisque, en effet, il est vrai), relatives à } \\
22,5 \%, \text { appositions à 7,5\%, et } \\
\text { marqueur ø à 30\%. }\end{array}$ & $\begin{array}{l}\text { Utilisation de marqueurs à } \\
18 \% \text { (since for hence) } 36 \% \text { de } \\
\text { relatives, marqueur } ø 46 \%\end{array}$ \\
\hline
\end{tabular}

Ce tableau chiffré, résumant l'utilisation des marqueurs de cause dans des discours politiques nationalistes, montre un usage décroissant de ceux-ci, de la catégorie la moins subjective (configuration 1) à la catégorie la plus subjective (configuration 4), si nous considérons que la relative n'est pas l'exact équivalent d'un marqueur causal (mais nous y reviendrons dans la dernière partie). Ces résultats sont comparables à ceux mis en évidence par le tableau 4, consacré à l'analyse du discours des blogs, même si les proportions ne sont pas les mêmes.

Il est selon nous intéressant que le marquage explicite de la relation causale ainsi s'estompe au profit de l'utilisation de la relative au fur et à mesure de la plus grande subjectivité des énoncés.

\section{Syndète/asyndète : quel rôle pour le marqueur dans la relation de cause?}

Ces tableaux synthétiques, qui mettent en évidence la relation entre une configuration argumentative et l'utilisation ou non d'un marqueur selon la langue, ne doivent pas nousfaire oublier que la relation syntaxique la plus fréquente dans la presse et dans les blogs est tout de même l'asyndète, et ce dans les deux langues. Nous posons donc le problème suivant : pour quelle raison un marqueur est-il nécessaire dans certains cas, puisqu'il nous est tout-à-fait possible de comprendre une relation d'effet à cause sans marque syntaxique? Ou, autrement dit : qu'apporte le marqueur à la construction du sens tant en anglais qu'en français?

Ce qui nous semble essentiel est que le marquage explicite de la détermination n'est pas seulement fonction de la relation entre le contenu de RP1 et le contenu de RP2, ni même de la relation énonciateur/contenu. Si nous constatons des variations d'un tableau contrastif à l'autre (presse, blogs, discours nationalistes), il est possible que ce soit en réalité la relation entre énonciateur et co-énonciateur qui forme un paramètre essentiel à la construction linguistique de la relation de cause.

\section{III.1. Construction de la relation de cause}

Dans la T.O.E, au niveau notionnel, la relation de cause fait partie des relations primitives: elle relève de la logique. Cela signifie que pour chaque fait, il est possible d'envisager un éventail d'explications acceptables par tout locuteur. Linguistiquement, pour une lexis $P$ permettant d'exprimer un fait nous avons un ensemble de lexis $Q$ susceptibles d'être utilisées par tout énonciateur. Néanmoins, une fois qu'une lexis $Q$ est choisie et mise en relation avec $\mathrm{P}$, c'est-à-dire une fois que l'énonciateur a sélectionné une 
cause parmi toutes les lexis explicatives possibles, le marqueur de lien indique au coénonciateur, au niveau énonciatif, la façon dont doivent être considérées les autres lexis possibles mais non sélectionnées par rapport à la lexis sélectionnée. Ceci implique que le marqueur n'a pas pour rôle l'explicitation de la relation de cause elle-même, compréhensible et possible avant la mise en relation, mais de la façon dont l'énonciateur considère la cause sélectionnée. Par exemple en (26) :

(26) Quant à la Turquie, Sarkozy trompe son monde comme il l'a fait sur la Constitution européenne. Il a laissé et même encouragé la commission Balladur sur la réforme des institutionsà supprimer le referendum obligatoire qui avait été inscrit dans la Constitution française à l'article 88-5 pour toute nouvelle adhésion à l'Union européenne après la Croatie. Il est vrai que MM. Kouchner et Jouyet, respectivement ministre des Affaires Etrangères et secrétaire d'Etat aux affaires européennes sont de chauds partisans de l'adhésion de la Turquie. JMLP.

$\mathrm{Au}$ niveau notionnel, il y a de nombreuses explications possibles à la lexis / Sarkozi, abandonner référendum/ parmi lesquelles nous pouvons proposer: /Français, être incapable de juger/ /être nécessaire de faire vite/ etc...

Au niveau prédicatif, nous avons :

RP1 <Sarkozi - abandonner -referendum> expliquée par RP2 <Kouchner et Jouyet - être partisan de l'adhésion de la Turquie>.

Le marquage explicite de cette relation par il est vrai que, au-delà de sa valeur causale et argumentative, indique au co-énonciateur la façon dont l'énonciateur envisage la cause sélectionnée par rapport à l'ensemble des possibles. Par conséquent, plus que le sens de l'énoncé, puisque la relation de cause peut être reconstituée sans marqueur, c'est, selon nous, la nature de la relation inter-énonciative qui va déterminer le choix d'un marqueur ou d'une structure particulière. Ce marqueur est ainsi la trace de la façon dont l'énonciateur désire présenter la cause qu'il a sélectionnée par rapport aux possibles au co-énonciateur.

51 Dans cet exemple, le marquage par il est vrai que indique que l'énonciateur présente la cause sélectionnée comme suffisante et éliminant d'office toutes les autres causes possibles : elle marque un retour sur un préconstruit ainsi qu'une complicité avec le lecteur.

Chaque marqueur permet ainsi au co-énonciateur de comprendre comment l'énonciateur considère la cause mentionnée par rapport aux causes possibles.

En revanche si nous enlevons le marqueur de cause de l'énoncé (26) :

(26') Quant à la Turquie, Sarkozy trompe son monde comme il l'a fait sur la Constitution européenne. Il a laissé et même encouragé la commission Balladur sur la réforme des institutionsà supprimer le referendum obligatoire qui avait été inscrit dans la Constitution française à l'article 88-5 pour toute nouvelle adhésion à l'Union européenne après la Croatie. ø MM. Kouchner et Jouyet, respectivement ministre des Affaires Etrangères et secrétaire d'Etat aux affaires européennes sont de chauds partisans de l'adhésion de la Turquie.

Il est difficile de savoir comment l'énonciateur considère la cause sélectionnée relativement à l'éventail des possibles. Notre hypothèse est que l'absence de marqueur indique que l'énonciateur ne souhaite pas que le co-énonciateur se réfère à une alternative. La cause présentée l'est de façon privilégiée. 
Pour revenir aux observations précédentes, il apparaît que la différence de marquage entre les genres de discours correspond à une relation différente entre énonciateur et coénonciateur, tant dans la langue que dans le genre.

\section{L'incidence des relations inter-énonciatives}

\section{IV.1. Le cas de la presse}

Dans la presse, la différence entre les deux langues est marquée, et nous obtenons un tableau en miroir, que nous rappelons ici :

Tableau 3 : Utilisation des marqueurs de cause dans la presse quotidienne

\begin{tabular}{|l|l|l|}
\hline Configuration argumentative & anglais & français \\
\hline 1. Explication factuelle portant sur un fait & $\begin{array}{l}50 \% \\
\text { Because }\end{array}$ & $11 \%$ \\
\hline 2. Explication modale portant sur un fait & $39 \%$ & $16 \%$ \\
\hline 3. Explication factuelle portant sur une modalité & $18 \%$ & $\begin{array}{l}22 \% \\
\text { En effet }\end{array}$ \\
\hline 4. Explication modale portant sur une modalité & Proche $0 \%$ & $\begin{array}{l}34 \% \\
\text { Car }\end{array}$ \\
\hline
\end{tabular}

Si l'utilisation d'un marqueur confirme un lien causal et permet au co-énonciateur de retracer la relation entre cause sélectionnée et causes possibles, est-ce à dire que lorsqu'il n'utilise pas de marqueur l'énonciateur laisse le co-énonciateur libre d'envisager la relation de cause ou pas ? En effet, Rossette (2005) suggère que le co-énonciateur pourrait être plus 'libre' (ou moins contraint) lorsque la relation de cause est marquée par une asyndète.

Selon nous, s'il existe une relation de cause à effet entre les deux lexis au niveau notionnel, alors le co-énonciateur l'établira à la lecture. Mais il ne saura pas comment l'énonciateur situe ou envisage cette cause par rapport aux possibles. C'est ce qui se passe en anglais lors de l'explication modale portant sur une modalité (configuration 4), ou en français lors de l'explication factuelle portant sur un fait (configuration 1), dans le corpus de presse.

Voici deux énoncés illustrant les tendances mises en évidence, l'un en anglais pour la configuration 4 et l'autre en français pour la configuration argumentative 1 :

(27) Never has the job of school leaders in articulating those values, day by day, week by week, been so important. $\varnothing$ For many children, school and its values, its clear boundaries and moral frame work are the only solid bedrock in their lives. The Guardian International.

(28) En tout, 8732 fonctionnaires de l'Etat fédéral travaillent encore dans l'ancienne capitale de l'Allemagne divisée, quand Berlin en compte 8931... Ce dédoublement 
fait hurler ceux qui traquent la dépense publique.ø Parmi le personnel gouvernemental, ils sont encore des centaines à faire la navette entre les deux villes plusieurs fois par semaine. Le Monde. méthode imposée par les paramètres de notre recherche (l'absence ou la présence de marqueur), et sont donc à prendre avec beaucoup de précautions. Il est cependant intéressant, si cela peut être confirmé par une étude sur un corpus plus important, que l'asyndète soit majoritairement utilisée en anglais dans les énoncés à valeur argumentative et dans les énoncés français à valeur explicative factuelle. Cela signifie que l'énonciateur ne souhaite pas que le co-énonciateur se réfère à l'ensemble des possibles lors d'une explication factuelle dans la presse en français et lors d'une argumentation dans la presse en anglais. L'alternative est possible, puisqu'au niveau notionnel l'éventail des causes existe qu'il soit mentionné ou pas. Mais dans les deux cas étudiés ci-dessus, l'énonciateur ne propose pas de s'y référer.

66 Il semble que, lorsqu'il n'y a pas de relation directe au co-énonciateur, et que donc la construction $\mathrm{du}$ discours se fait dans une relation inter-énonciative abstraite, le raisonnement se construise de façon opposée en français et en anglais. Ceci est, selon nous, l'indice d'une prise en compte du co-énonciateur totalement différente : celui-ci peut remonter à des alternatives possibles pour un raisonnement factuel en anglais et pour un raisonnement teinté de modalité en français. 


\section{IV.2. Le cas des blogs}

Dans les blogs, dans une lecture de haut en bas du tableau 4 on passe, dans les deux langues, d'un marquage explicite à un marquage totalement implicite et asyndétique.

Tableau 4 : Présentation synthétique du classement des énoncés extraits des blogs de journalistes

\begin{tabular}{|c|c|c|}
\hline Configuration argumentative & Anglais & Français \\
\hline $\begin{array}{l}\text { 1.Explication factuelle portant } \\
\text { sur un fait }\end{array}$ & 50 \%marqueurs Because $35 \%$ & $\begin{array}{l}53 \% \text { marqueurs } \\
\text { car et parce que }\end{array}$ \\
\hline $\begin{array}{l}\text { 2.Explication modale portant } \\
\text { sur un fait }\end{array}$ & $\begin{array}{l}60 \% \text { marqueur , } 25 \% \text { because ou } \\
\text { forme de questions en RP1 }\end{array}$ & $\begin{array}{l}58 \% \text { marqueurs } \\
\text { Car, parce que, en effet, } \\
\text { quelques relatives }\end{array}$ \\
\hline $\begin{array}{l}\text { 3.Explication factuelle portant } \\
\text { sur une modalité }\end{array}$ & $30 \%$ marqueurs, relative & $50 \%$ marqueurs \\
\hline $\begin{array}{l}\text { 4.Explication modale portant } \\
\text { sur une modalité }\end{array}$ & $10 \%$ marqueurs & $-39 \%$ marqueurs \\
\hline
\end{tabular}

Ce tableau montre une moindre utilisation des marqueurs dans les deux dernières configurations dans les deux langues. Ainsi, plus la relation de cause est déterminée de façon modalisée, moins l'énonciateur est susceptible d'utiliser un marqueur, et ceci que ce soit en anglais ou en français. Il apparait également une disparité prononcée entre les deux langues : en configurations 3 et 4 , les proportions sont bien moindres en anglais.

Observons plus précisément la construction du sens causal dans des énoncés classés en configurations 1 et 4 .

\section{Configuration 1}

En anglais :

(29) Not just the Bullingdon Club, the fox hunting, the family fortunes, and the heart-wrenching stories of his son Ivan, but what really makes this man tick.

We need to know because it is the emotional makeup of the man which has shaped the governments of his two immediate predecessors. Tony Blair's remarkable ability to convince himself of his own sincerity, Gordon Brown's inner demons of self-doubt and fearfulness : this is the stuff which has determined Labour's political fortunes in an age when ideas and policyhave so much less traction on the political process.Madeleine Bunting.

En français :

(30) L'homme d'Allianz insiste et leur explique : ce n'était pas une émeute stricto sensu car elle n'était pas dirigée contre le gouvernement, c'était un mouvement de foule.

En français comme en anglais, la relation est marquée explicitement. Nous retrouvons la structure relevée dans la presse anglophone pour cette configuration. En revanche, en français, les chiffres sont beaucoup plus importants dans les blogs que dans la presse. Comme si la cause factuelle était plus facilement proposée à la discussion en français dans 
les blogs que dans la presse. L'alternative à la cause sélectionnée ou nécessaire reste envisageable. Si nous manipulons l'énoncé français :

(30') L'homme d'Allianz insiste et leur explique : ce n'était pas une émeute stricto sensu, $\varnothing$ elle n'était pas dirigée contre le gouvernement, c'était un mouvement de foule.

Il semble que dans ce cas nous perdions la caractéristique factuelle de la cause nécessaire, et la préconstruction de la relation. En effet, (30) fait partie d'un raisonnement élaboré par un assureur à partir d'un texte de loi. Il s'agit donc de tracer un chemin vers une cause préétablie indépendamment de l'énonciateur ou du locuteur, cause à la disposition de quiconque en aurait besoin. Sans le marqueur en (30'), l'énoncé se rapproche d'un raisonnement personnel, sans pré-construction extérieure à l'énonciateur. Il semble qu'il ne soit pas fait de place à l'alternative, l'énonciateur propose une cause qu'il privilégie et ne souhaite pas que le co-énonciateur se réfère à l'éventail des possibles.

La relation syntaxique marquée serait pour l'énonciateur une façon de signifier au coénonciateur que son raisonnement est préconstruit. Elle ne contribuerait donc pas à la construction du sens de la relation causale, mais à celle du sens du raisonnement.

Manipulation de l'énoncé (anglais) :

(29') Not just the Bullingdon Club, the fox hunting, the family fortunes, and the heart-wrenching stories of his son Ivan, but what really makes this man tick.

We need to know $\varnothing$ it is the emotional makeup of the man which has shaped the governments of his two immediate predecessors. Tony Blair's remarkable ability to convince himself of his own sincerity, Gordon Brown's inner demons of self-doubt and fearfulness : this is the stuff which has determined Labour's political fortunes in an age when ideas and policy have so much less traction on the political process. (Madeleine Bunting)

En (29), la relation syntaxique ne peut être transformée ainsi. Nous devons ajouter une marque de ponctuation forte, point ou tiret en anglais. Le fait d'utiliser because permet selon nous à l'énonciateur de présenter son raisonnement comme retraçable et assorti d'alternatives possibles qu'il a envisagées. En (29'), énoncé dans lequel nous avons supprimé because, la relation est présentée comme le résultat d'un raisonnement personnel, sans prise en compte de la part de l'énonciateur d'une possible préconstruction reconnaissable par le co-énonciateur.

\section{Configuration 4}

75 En configuration 4, avec l'explication d'un énoncé à valeur modale par un autre énoncé à valeur modale, il est plus rare que dans les autres configurations de relever des marqueurs. Comme dans la presse anglophone. Cependant, la proportion d'énoncés construits à l'aide d'un marqueur est beaucoup moins importante en anglais. Cela signifierait, selon notre hypothèse, que l'énonciateur renvoie plus aisément à l'éventail des causes possibles en français qu'en anglais, et donc que, dans cette catégorie plus spécifiquement argumentative, l'énonciateur français présente plus fréquemment la relation de cause comme préconstruite et assortie d'alternatives. Mais globalement, c'est dans la partie argumentative du discours des blogs, tant en français qu'en anglais, que l'énonciateur se passe le plus facilement des marqueurs.

En français :

(31) Dans un entretien au Parisien, il explique qu'il se sacrifie pour "pacifier" une pré-campagne qu'il juge "extrêmement violente". Il ne vise personne, mais on devine que son regard se tourne vers Roger Karoutchi. 
Depuis le début de l'été, ce dernier s'emploie fébrilement à démontrer qu'il est le candidat naturel de l'UMP. Il passe des centaines de coups de fil, bombarde de mail les militants qui seront appelés, en mars 2009, à choisir le candidat de l'UMP. (Blog d'Alain Auffray).

En français, dans l'énoncé (31) la relation de cause porte sur la séquence on devine que son regard se tourne vers Roger Karoutchi. Et plus particulièrement sur on devine. Il nous semble que l'asyndète se justifie par le fait que la cause invoquée n'est pas sujette à discussion. L'énonciateur veut présenter cette cause précisément et ne propose donc pas de possibilité de référence à un éventail de causes.

En anglais :

(32) They know I'm Jewish. They're wondering if I might like to write about, maybe even get a little bit involved with, something called the Jewish Community center for London.

And no, I wouldn't, really. Fine, this community centre has just got planning permission, it's costing a lot, it'll be a beautiful building near Hampstead, it's going to open in 2013 and it'll have amazing activities for people to celebrate their heritage and all that. But it's all rather earnest -a bit, I don't know, Jewish for me. The truth is, and this is probably a bad thing, but I'd be more excited if a new Apple Store were opening in my town. (Blog de Jonathan Magolis)

En anglais, nous avons $\mathrm{P}$, construit à partir de RP1 <person - write - Jewish Community Center in London>expliqué par $\mathrm{Q}$, construit à partir de RP2 < Jewish Community Center in London - be - earnest >. Le fait de ne pas utiliser de marqueur pour mettre les deux propositions en relation indique selon nous que l'énonciateur ne désire pas que le coénonciateur se réfère à l'éventail des causes possibles. Il singularise la cause qu'il avance, sans ouverture à la discussion. Il est d'autre part intéressant de constater la singularité de la cause sélectionnée : But it's all rather earnest - a bit, I don't know, Jewish for me.

Cette dernière configuration - explication portant sur une modalité - correspond à des énoncés teintés d'une forte ironie, en anglais comme en français. Peut-être l'ironie, pour être perçue comme telle, ne doit-elle pas permettre au co-énonciateur de revenir sur le choix effectué parmi les causes possibles. La relation doit être exclusive de toute autre, même si énonciateur et co-énonciateur savent que l'alternative existe. Soient les énoncés ci-dessous :

(33) Christine Albanel est, elle aussi, héroïque. Une fromagerie, un élevage ovin, ... Quel rapport avec la culture? La ministre se serait bien passée de ce détour par la Champagne humide. Elle doit être le soir même à Cannes pour le salon international des programmes audiovisuels. (Blog d'Alain Auffray)

(34) So don't tell me that being Jew-ish rather than a full-on Jew is a cowardly copout. As far as avoiding antisemitism is concerned, being merely and meekly Jew-ish doesn't help at all. (Blog de John Magolis)

Il semble que le sens de l'ironie se construise, en partie, sur l'asyndète, en anglais comme en français. En effet, cette structure met en relief un rapport entre effet et cause qui échappe à la préconstruction.

En (33), P, construit à partir de RP1 : <Christine Alabanel - être -héroïque> est expliquée par $\mathrm{Q}$, construite à partir de RP2 <fromagerie/élevage ovin -avoir - rapport avec la culture> Le terme héroïque sur lequel porte l'explication est disproportionné par rapport à la cause introduite,d'où un effet comique. Il n'est pas possible pour le co-énonciateur de préconstruire un ensemble de causes à l'héroïsme de Christine Albanel qui inclue la cause invoquée par l'énonciateur. Le fait qu'il n'y ait pas de relateur entre les deux propositions indique au co-énonciateur que l'énonciateur ne propose pas de rapport à d'autres causes 
possibles. Il la lui propose comme une cause unique, non-discutable, alors que le coénonciateur sait qu'il peut y avoir d'autres justifications à l'héroïsme.

En (34) le co-énonciateur pourrait commencer à élaborer l'ensemble des raisons qui justifient la forme négative de RP1 < being Jewish - be - cop-out > ou l'interdiction de le considérer ainsi : ces justifications appartiendraient au domaine notionnel du courage. Mais la justification invoquée n'a rien à voir avec le courage, seulement avec le danger.

Nous pouvons manipuler ces deux énoncés :

(33') Christine Albanel est, elle aussi, héroïque. Une fromagerie, un élevage ovin, ... En effet, quel rapport avec la culture? La ministre se serait bien passée de ce détour par la Champagne humide. Elle doit être le soir même à Cannes pour le salon international des programmes audiovisuels.

(34') So don't tell me that being Jew-ish rather than a full-on Jew is a cowardly copout. Because as far as avoiding antisemitism is concerned, being merely and meekly Jew-ish doesn't help at all.

Avec l'introduction de marqueurs de cause, nous basculons dans le registre du raisonnement induisant la prise en compte d'un chemin vers des alternatives possibles. L'effet comique, il nous semble, disparaît, car nous perdons la construction ironique, peut-être en raison du caractère appuyé, explicite de la démonstration, qui fait que la justification invoquée est incluse dans un éventail de possibles.

D'après ces exemples, représentatifs de cette partie de notre corpus, l'ironie se construit de la même façon en anglais et en français.

Le fait de ne pas retrouver de disparité aussi grande dans le marquage syntaxique que celle observée dans le discours journalistique, montre d'une part qu'il est difficile en linguistique contrastive de parler de l'anglais et du français de façon globale. D'autre part, la relation inter-énonciative des blogs, moins encadrée et permettant une réponse, fait que le marquage syntaxique de la cause est assez similaire en anglais et en français. Est-ce la présence potentielle d'un co-énonciateur inter-actif qui induit une syntaxe différente en français de celle utilisée dans la presse ? Le sens construit autour de - ou sur - la cause par l'asyndète ou les marqueurs est comparable en anglais et en français.

\section{IV.3. Le cas des discours politiques nationalistes}

Dans les discours politiques à tendance nationaliste ici, l'absence de marqueur, ou l'asyndète, est beaucoup moins fréquente que dans les deux types de discours présentés précédemment. Le choix des marqueurs eux-mêmes est également intéressant, parce que, que ce soit en anglais ou en français, on observe des spécialisations selon les configurations argumentatives (cf. Tableau 5 ci-dessous).

Tableau 6 : Synthèse du classement des énoncés extraits des discours politiques nationalistes

\begin{tabular}{|l|l|l|}
\hline $\begin{array}{l}\text { Configuration } \\
\text { argumentative }\end{array}$ & Français & Anglais \\
\hline $\begin{array}{l}\text { Explication factuelle } \\
\text { portant sur un fait } \\
\text { configuration 1 }\end{array}$ & $\begin{array}{l}\text { Utilisation de marqueurs à } 60 \% .30 \% \\
\text { de participes présents et 1\% de } \\
\text { participes passés. } \\
\text { Asyndète quasi nulle. }\end{array}$ & $\begin{array}{l}\text { Utilisation de marqueurs à } \\
83 \% \text { because, as, after. }\end{array}$ \\
\hline
\end{tabular}




\begin{tabular}{|c|c|c|}
\hline $\begin{array}{l}\text { Explication factuelle } \\
\text { portant sur une modalité } \\
\text { configuration } 2\end{array}$ & $\begin{array}{l}\text { Utilisation de marqueurs à } 96 \% \\
\text { parce que, car et le verbe expliquer dans } \\
\text { différentes locutions verbales. }\end{array}$ & $\begin{array}{l}\text { Utilisation de marqueurs à } \\
80 \% \text {, because, as. }\end{array}$ \\
\hline $\begin{array}{l}\text { Explication modale } \\
\text { portant sur un fait } \\
\text { configuration } 3\end{array}$ & $\begin{array}{l}\text { Utilisation de marqueurs à } 30 \% \\
\text { puisque, en effet. }\end{array}$ & $\begin{array}{l}\text { Utilisation de marqueurs à } \\
17 \% \\
\text { because }\end{array}$ \\
\hline $\begin{array}{l}\text { Explication modale } \\
\text { portant sur une modalité } \\
\text { configuration } 4\end{array}$ & $\begin{array}{l}\text { Utilisation de marqueurs à } 40 \% \text { ( } \\
\text { puisque, en effet, il est vrai), relatives à } \\
22,5 \% \text {, appositions à } 7,5 \% \text {, et } \\
\text { marqueur } \varnothing \text { à } 30 \% \text {. }\end{array}$ & $\begin{array}{l}\text { Utilisation de marqueurs à } \\
18 \% \text { (since for hence) } 36 \% \text { de } \\
\text { relatives, marqueur } \varnothing 46 \%\end{array}$ \\
\hline
\end{tabular}

87 Nous nous intéresserons, dans ce corpus, essentiellement à l'explication portant sur une modalité, configuration argumentative dans laquelle nous avons relevé une très large proportion d'énoncés utilisant des propositions relatives.

\section{Etude de cas : la structuration des relations de cause par une relative dans les discours nationalistes politiques}

La relation inter-énonciative transparaît dans la structure utilisée - une relative ici pour exprimer la cause : l'objectif de l'énonciateur/locuteur est selon nous de faire en sorte que, grâce à l'introduction avec la relative d'un commentaire qui s'inscrit dans une démarche causale-explicative ${ }^{1}$, l'auditoire se constitue en groupe mis en danger, sans place offerte à l'argumentation.

\section{V.1. Marquage par une relative en anglais}

89 Soient les énoncés suivants, dans lesquels nous pourrions substituer à la relative une subordonnée de cause introduite par because ou parce que :

(35) We will abolish the 'positive discrimination' schemes that have made white Britons second-class citizens. NG.

(36) The BNP will crack down on crime and restore public safety and confidence. We will free the police and courts from the politically correct straitjacket that is stopping them from doing their job properly. NG.

(37) We are against the 'trendy' teaching methods that have made Britain one of the most poorly educated nations in Europe. NG.

Dans les énoncés (35) à (37), l'un des termes de P - the 'positive discrimination' schemes/ the politically correct straitjacket/ the 'trendy' teaching methods - est qualifié par la relative qui tient lieu de proposition $Q$ introduite par that. Cette qualification a une valeur péjorative : poorly educated (...), second-class citizens, that is stopping them from doing their jobs.

91 D'après G. Mélis², l'introduction d'une proposition relative en that, détachée, c'est-à-dire précédée d'une virgule, apporte une valeur contrastive définitoire qui permet la définition d'une occurrence. 

straitjacket, the 'trendy' teaching methods- qui se voient attribuer une propriété définitoire - that have made white Britons second-class citizens, that is stopping them from doing their job properly, that have made Britain one of the most poorly educated nations in Europe. Cette propriété définitoire permet de contraster le référent de l'antécédent (globalement, des mesures politiques) à ce que le locuteur lui-même proposerait.

Dans la construction du discours, le commentaire à valeur péjorative porte le plus souvent sur l'auditoire (poorly educated (...), second-class citizens, that is stopping them from doing their jobs ) et lui permet de se constituer en groupe nation mis en danger, groupe nation-auditoire qui se construit en opposition à tout groupe extérieur.

\section{V.1.1. Construction du sens de la relative}

d'ouverture à l'argumentation, dans la mesure où la structure utilisée n'est pas d'emblée sémantiquement associée à la relation d'effet à cause. D'autre part, c'est le contenu de $\mathrm{P}$ qui permet d'interpréter ou de substituer à la relative une circonstancielle de cause :

- (35) RP1 <BNP - abolish - positive discrimination scheme ] because RP2 [positive discrimination scheme - make - white briton ...>

- (36) RP1<BNP- free - police and court from the politically correct straitjacket] because RP2 [ politically correct straitjacket -stop - police from ...>

- (37) RP1<BNP- be against - trendy teaching method >because RP2 < trendy teaching method - make - british people poorly educated >

Nous constatons dans ces schématisations que c'est la valeur modale des verbes de $\mathrm{P}$ abolish, free, be against, ajoutée à la détermination par le modal will, qui amène le coénonciateur à donner une valeur causale à la relative. L'énonciateur, ici locuteur du discours à la première personne, s'engage à agir, proposition $\mathrm{P}$, et qualifie péjorativement l'élément sur lequel il s'engage à agir en $Q$. Qualification modale qui prend une valeur explicative relativementà l'action promise en $P$.

Ces énoncés ont été relevés dans la partie la plus subjective du discours, donc celle où il est proposé une argumentation personnelle, et paradoxalement cette structure, n'offre pas d'ouverture à la discussion. L'énonciateur construisant une qualification, le chemin vers l'éventail des causes au niveau notionnel n'existe pas.

Cette façon de construire la relation causale fonctionnerait-elle avec un énoncé nonmodal? Même dans les autres genres de discours, nous n'avons relevé de relatives à valeur explicative que dans les deux configurations argumentatives dans lesquelles l'explication porte sur un énoncé à valeur modale. Nous présentons ci-dessous un énoncé relevé dans un blog:

(38) Sur l'histoire, dont on sait qu'elle n'est jamais que l'ombre du squelette d'un roman, il ne faut jamais trop en dire, non seulement pour ne pas gâcher aux autres la marche de la lecture, mais encore parce que l'intrigue n'est jamais l'essentiel.(Blog de P. Assouline) 


\section{V.2. Marquage par une relative en français}

(39) Le Front National n'a eu de cesse de dénoncer ce système destructeur de richesses, qui au final, mutualise les pertes et privatise les profits, à l'instar de l'affaire du« Crédit Lyonnais ». JMLP.

(40) La ratification du traité de Lisbonne ne fait que renforcer le statut "d'indépendance" de la Banque Centrale Européenne, mission effectuée par N. Sarkozy pour le compte de l'oligarchie financière qui l'a porté au pouvoir et qui parachève le programme européiste initié par le traité de Maastricht en 1992. JMLP.

(41) Vous le voyez, mesdames et messieurs, le Front national entend se donner les moyens de restaurer les pouvoirs régaliens de l'Etat, pour disposer des marges de manœuvre nécessaires au redressement de la France, et combattre l'équation ANTISOCIALE de l'Europe de Maastricht, de Francfort, de Lisbonne ... et de Washington, qui asservit les Français. JMLP.

En français, notons également la qualification du référent de l'antécédent avec une valeur péjorative. Ce référent cependant, est moins systématiquement identifiable à l'auditoire. Avec les énoncés français, nous avons l'introduction d'un commentaire grâce à la qualification/définition de la source d'un malaise autour duquel peut se construire l'identité de l'auditoire en groupe 'nation' :

- En (39) mutualise les pertes etc.

- En (40) parachève le programme européiste avec valeur modale particulière de cet adjectif.

- En (41), même si c'est moins systématique qu'en anglais : qui asservit les français, indique la prise en compte du groupe nation auquel peut s'identifier l'auditoire.

Si nous détaillons la construction des énoncés de (39) à (41) :

- En (39) RP1 <Front National - dénoncer - système destructeur de richesse] parce que RP2 [ système destructeur de richesse - mutualiser-perte > et RP2' <système destructeur de richesse- privatiser - profit>

- En (40) RP1 <N. Sarkozy -effectuer - mission pour oligarchie financière>parce que RP2 $<$ oligarchie financière - porter au pouvoir- N. Sarkozy>

- En (41) RP1 <Front National - combattre - équation ANTI-SOCIALE> parce que RP2 <équation ANTI-SOCIALE - asservir - Français>

99 Il apparaît que la modalité introduite en RP1 pour construire P est expliquée par une modalité introduite en RP2 pour construire Q. La construction du sens causal est entièrement fondée sur la modalité. La relative, proposition $Q$, qualifie l'élément polémique dans $\mathrm{P}$, et met en relief la propriété qui en fait un élément polémique. Cette propriété étant construite sur une valeur modale, elle relève de l'entière responsabilité de l'énonciateur. Le co-énonciateur est cependant à-même de reconstruire la valeur causale de la relative, il y adhèrera ou pas.

Nous constatons donc qu'en anglais comme en français la relative prend un sens causal dans une partie très modale du discours. C'est en tout cas dans ces parties que nous avons relevé une majorité de nos relatives-explicatives.

Il semble qu'avec cette façon de structurer la relation de cause, et donc en ne renvoyant pas à l'ensemble des causes possibles, l'argumentation soit fermée: le co-énonciateur adhère ou pas. 


\section{Conclusion}

Nous avons étudié principalement trois structures syntaxiques susceptibles de marquer une relation de cause : marqueur explicite, asyndète, relative.

Nous n'avons pas noté une proportion égale de l'utilisation de ces structures en fonction des différents types de discours, des configurations argumentatives et de la langue. Nous observons au contraire :

- Des utilisations inversement proportionnelles des marqueurs en anglais et en français dans la presse.

- Une utilisation comparable de l'asyndète et des marqueurs en anglais et en français dans les blogs selon les configurations argumentatives, avec une prééminence de l'asyndète dans les configurations argumentatives à valeur modale.

- Une utilisation comparable en anglais et en français dans les discours politiques à tendance nationaliste avec une prééminence des relatives dans la configuration argumentative à valeur modale.

04 L'asyndète correspond selon nous à une mise en relation qui est exclusive de toute autre possibilité malgré l'éventail des possibles. L'énonciateur n'envisage pas la contradiction. Nous l'avons relevée en plus grande proportion dans la configuration argumentative modale en anglais et dans la configuration factuelle en français dans la presse, puis dans les configurations modales dans les blogs et les discours politiques à tendance nationaliste en anglais et en français. Il semble donc que l'on ne puisse pas faire correspondre un marqueur à un autre de façon systématique sans tenir compte du type de discours lors de la traduction. Nous constatons également une singularisation du mode argumentatif dans la presse française, sans exposition explicite de raisonnement lors de la mise en relation causale factuelle. L'utilisation presqu'exclusive de l'asyndète dans les énoncés à valeur ironique dans les blogs tant en anglais qu'en français tend à montrer que l'énonciateur élimine ostensiblement toute explication alternative au profit d'une complicité avec le co-énonciateur, complicité souvent fondée sur l'incongruité de la mise en relation proposée.

5 Dans une relation causale, le marqueur renvoie à l'éventail des possibles. Il s'agit donc d'une argumentation ouverte, à travers laquelle la position de l'énonciateur est accessible au co-énonciateur. D'après nos chiffres, cette argumentation ouverte caractérise la configuration factuelle dans la presse en anglais et la configuration modale en français, puis les configurations factuelles des blogs et discours politiques à tendance nationaliste en anglais et en français. Tout se passe comme si la relation à un co-énonciateur plus distant dans la presse impliquait en français une utilisation différente des structures syntaxiques, comme si le sens construit autour de la relation de cause devait se faire à l'inverse de ce qu'il est dans une relation intersubjective plus directe.

La relative ne peut, selon nous, renvoyer à l'ensemble des possibles. L'échange argumentatif n'est donc pas proposé. Il s'agit d'une polémique en forme de oui ou non. Le co-énonciateur adhère ou pas à la cause invoquée par l'énonciateur. L'énonciateur s'adresse à un auditoire acquis à sa cause.

Nous proposons, pour résumer, les schémas suivants : 


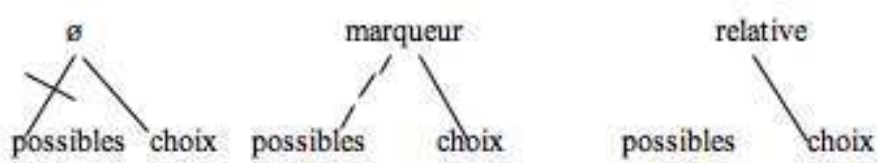

La structure syntaxique propose un mode d'argumentation en fonction d'un type de discours dans un système linguistique donné. Elle ne permet pas de construire le sens de la relation de cause qui, faisant partie des relations dites primitives, n'a pas besoin de marqueur pour être comprise.

Les structures syntaxiques que nous avons étudiées permettent de construire du sens autour de la relation de cause, structure qui peut varier en fonction de la relation inter énonciative, ou de la langue pour une relation inter-énonciative identique (cas de la presse).

\section{BIBLIOGRAPHIE}

ANSCOMBRE, J-C. (1984) «La représentation de la notion de cause dans la langue », Cahiers de grammaire, $8: 4-53$.

BAKER, M. (1998) « Réexplorer la langue de la traduction : une approche par corpus ». META, XLIII, $4: 480-485$.

BENVENISTE, É. (1970), «L'appareil Formel de l'Enonciation », Problèmes de Linguistique Générale, Gallimard, pp79-88.

CELLE, A. (2006) Temps et modalité. L'anglais, le français et l'allemand en contraste. In A.-M. LAURIAN \& T. szende (éd.), Etudes Contrastives, vol. 7, Bern, Berlin, Bruxelles, Frankfurt am Main, New York, Oxford, Wien, Peter Lang.

CoSME, C. (2006) « Clause combining across languages. A corpus-based study of English-French translation shifts ", Languages in Contrast, $6: 1$, John Benjamins Publishing Company, 71-108.

DE COLA-SEKALI, M. (1991) Connexion inter-énoncés et structuration des relations temporelles et argumentatives en anglais contemporain. Une étude énonciative des connecteurs polyvalents since et for, Thèse de doctorat, Université Paris 3 - Sorbonne Nouvelle.

DELECHELLE, G. (1994) «Relations inter-énoncés de la subordination à la coordination, ou il y a coordination et coordination », RANAM, 27 :29-41.

DELECHELLE, G. (1989) L'expression de la Cause en Anglais Contemporain, Etude de quelques connecteurs et opérations, Thèse de doctorat d'état, Atelier National de reproduction des Thèses, Université Charles de Gaulle-Lille 3.

DELPORT, M-F. et CHEVALIER, J-C. (1995) L'Horlogerie de Saint-Jérôme. Problèmes linguistiques de la traduction, Paris, L'Harmattan.

DUCRot, O. (1983) « Puisque, essai de description polyphonique », Revue Romane, 24 :166-185. 
FLEISCHMAN, S. (1982) The Future in Thought and Language. Diachronic Evidence from Romance, Cambridge, Cambridge University Press.

GOUGENHEIM, G.[1929] (1971) Etude sur les périphrases verbales de la langue française, Paris, A. G. Nizet. GRANGER, S. (2003) « The corpus approach : a common way forward for Contrastive Linguistics and Translation Studies? ». In S. GRANGER, J. LEROT \& S. PETCH-TYSON (éds.), Corpus-based Approaches to Contrastive Linguistics and Translation Studies, Amsterdam, New York, Rodopi, 17-29.

GRIZE, J-B. (1997) Logique et langage, Paris, Gap, Ophrys.

GUIDERE, M. (2008) Introduction à la traductologie. Penser la traductologie : hier, aujourd'hui, demain, Bruxelles, De Boeck.

GUILLEMIN-FLESCHER, J. [1981] (1986) Syntaxe comparée du français et de l'anglais. Problèmes de traduction, Paris, Gap, Ophrys.

GUILLEMIN-FLESCHER, J. (1984) «Traduire l'inattestable », Cahiers Charles V, tome 6 « Linguistique comparée et Traduction : le statut modal de l'énoncé », Université Paris VII, 131-151.

GUILLEMIN-FLESCHER, J. (1996) «La traduction humaine : contraintes et corpus », Revue française de linguistique appliquée, vol. 1-2, 43-56.

HAMON, S. (2005) La Phrase Double Causale, Propriétés syntaxiques et interprétations sémantiques, Thèse de doctorat, Paris X-Nanterre.

HANOTE, S. (2000) Opérations énonciatives et représentation du discours dans le récit en anglais contemporain, Thèse de doctorat, Université de Poitiers.

JOHANSSON, S. (2003), « Contrastive linguistics and corpora ». In S. GRANGER, J. LEROT \& S. PETCHTYSON (éds.), Corpus-based Approaches to Contrastive Linguistics and Translation Studies, Amsterdam, New York, Rodopi, 31-44.

LANSARI, L. (2006) Les périphrases aller + inf. et be going to en français et en anglais contemporains, Thèse de doctorat, Université de Poitiers.

LARREYA, P. \& RIVIERE, C. [1991] (1999) Grammaire explicative de l'anglais, London, Harlow.

LEROUX, A (2009) « La citation comme argument dans la presse anglo-saxonne et dans la presse française ». In Alicja Kacprzak (éd.), Aspects interculturels de la communication,Presses de l'Université de Varsovie, 177-189.

LEROUX, A. (2009) «Une étude contrastive des relations causales dans les textes de presse en anglais et en français ». In J. Kortas, J. Jereczek-Lipinska, G. Quentel, (eds) La Globalisation Communicationnelle: Enrichissement et Menace pour les Langues, publications de l'Université de Gdansk.

LEROUX, A. (2010) «Le mode argumentatif dans le discours politique nationaliste, une étude de cas contrastive anglais/français : les discours de JM. Le Pen et de N.Griffin ». In Greta Komur Thilloy et Agnès Celles (eds) Le Discours Nationaliste en Europe, L'Improviste, Millau, 301-315.

NITA, R. (2007) « ironise un professeur vs. says a teacher, ou Sur les dérives du discours direct dans la traduction des verbes introducteurs - Etude contrastive français, anglais, roumain ». In C. DELESSE (éd.), Discours rapporté(s), Approche(s) linguistique(s) et/ou traductologique(s), Artois Presses Univeristé, 173-195.

OLSEN, M. (2006) « La construction logique de la fiction : les connecteurs », XVIe Congrès des Romanistes Scandinaves, Copenhague, Roskilde 2005, 
http://www.ruc.dk/cuid/publikationer/publikationer/XVI-SRK-Pub/MOL/

QUIRK, R. et al.(1985) A Comprehensive Grammar of the English Language, London, Longman.

ROSSETTE, F. (2005) « Parataxe et expressivité », in G. GIRARD-GILLET (éd.), Parcours linguistiques,

Domaine anglais, Travaux du CIEREC, 122 :245-257.

SALKIE, R. (2004) « Towards a non-unitary analysis of modality », in L. GOURNAY \& J.-M. MERLE

(éds.), Contrastes, Paris, Gap, Ophrys, 169-182.

WYLD, H. (2001) Subordination et Enonciation, Paris, Gap, Ophrys.

\section{NOTES}

1. Voir J. Guillemin-Flescher (1981), Syntaxe comparée du français et de l'anglais - problèmes de traduction, Gap, Ophrys. [GF]

2. Gérard Mélis, «Relatives et types de qualification », Cycnos, Volume 17 n Spécial, mis en ligne le 19 septembre 2008, URL : http://revel.unice.fr/cycnos

\section{RÉSUMÉS}

Dans cet article, nous présentons une synthèse de plusieurs études menées sur deux années, dans le cadre de la Théorie des Opérations Prédicatives et Enonciatives. Nous avons travaillé sur l'expression de la relation de cause en anglais et en français à travers des types de discours différents: la presse, les blogs de journalistes et les discours politiques nationalistes. La motivation première de ces analyses était de comprendre pourquoi il est finalement assez rare que nous utilisions un marqueur de cause pour exprimer la cause, tant en anglais qu'en français. Nous avons classé les énoncés constitués de deux propositions unies par une relation d'effet à cause en fonction de la portée de la relation causale (explication portant sur un fait, une propriété, etc.). Il est apparu que, d'une part, ce paramètre de portée est très important dans le marquage ou non-marquage de la relation de cause, avec une différence nette selon les langues, et que d'autre part, l'incidence du paramètre de portée, aussi appelé configuration causale, pouvait être modifiée en fonction du type de discours dans lequel se réalise la relation causale.

In this article I confront the results of three different studies which I carried out over the last two years: I observed the way English and French native speakers use causal conjunctions in three different types of discourse - journalistic written discourse, journalists' blogs or posts, and nationalist political speeches. My questioning originated in the fact that we scarcely use causal conjunctions to express cause. The corpus under study gathers authentic causal utterances in both languages rather than translations, and was classified using the parameter of scope, i.e. the nature of the element the causal utterance bears on : explanation of a fact, of a property, of a modality, etc. Results show that the overt use of a conjunction is subjected to the scope parameter and that juxtaposition of two clauses (asyndetic relation) does not correspond to the same scope in English and French. More significantly still, the contrastive grids (English/ French) were quite different from one type of discourse to the other, implying that the type of 
inter-personal relations involved in various types of discourse also has an incidence on the way causal relations are built

INDEX

Mots-clés : argumentation, asyndète, configurations argumentatives, corpus comparables, linguistique contrastive

Keywords : asyndeton, cross-linguistic studies, genre

\section{AUTEUR}

AGNÈS LEROUX

Université Paris Ouest - Nanterre 